ORAL INFECTION; CARDIOLOGY

\section{Oral health indicators poorly predict coronary heart disease deaths}

Tuominen R, Reunanen A et al. J Dent Res 2003; 82: 713-718

Associations between oral disease and heart disease deaths were explained by confounding factors and not by causation.

In recent years there has been mounting evidence against any causal role for oral factors in cardiovascular disease. In this Finnish study started in 1978-80, 6527 men and women were followed up for a mean 12 yrs after dental examination of caries. periodontal disease, other oral lesions and prostheses. Over the study period, causes of mortality were ascertained from the national register.

Death from coronary artery disease was significantly associated with the major cardiovascular risk factors: age, hypertension, hypercholesterolaemia, smoking, diabetes and educational level.

There were some significant associations between these factors and numbers of teeth, carious or filled teeth, retained roots, periodontal pockets and dental attendance. In unadjusted analysis, these oral factors were associated in some cases with the risk of death from heart disease. When adjusted for the known major factors, there was no effect on the risk of death from any oral factor.

doi:10.1038/sj.bdj.4810661

ALCOHOLISM; SUBSTANCE ABUSE; PERIODONTOLOGY

The influence of gingival margin recession on loss of clinical attachment in alcoholdependent patients without medical disorders

Khocht A, Janal M et al. J Periodonto/2003; 74: 485-493

Higher levels of alcohol abuse appeared to increase attachment loss in this study.

In this study, a group of 40 alcoholics, of whom 30 abused cocaine, was compared with a matched group of 25 non-alcoholics, of whom 14 abused cocaine. Groups were similar in mean age (40-42 yrs), gender, smoking and cocaine abuse. They differed in alcohol use (alcoholics about 4 times as much) and gamma glutamyl transpeptidase blood levels (GGTP; alcoholics 3 times higher).

There was no difference between groups in probing depth, attachment level (AL), recession, gingival inflammation, plaque amounts and number of teeth. However, with further analysis the investigators considered they had identified an effect, primarily in alcoholic subjects, where higher GGTP levels (>51 iu/l) were associated with higher plaque levels. Subjects above this level of GGTP had a mean $4 \mathrm{~mm} \mathrm{AL}$, and those below, $3 \mathrm{~mm}$. No association was found between cocaine use and AL.

\section{IMPLANT DENTISTRY}

\section{Crestal bone loss proximal to oral implants in older and younger adults}

\section{Bryant SR, Zarb GA J Prosthet Dent 2003; 89: 589-597}

Implants in patients aged 60-74 yrs did not lose bone any quicker than matched implants in patients aged 29-49 yrs.

There is limited published information on the effects of age on implant support. In this study, 35 complete prostheses (23 fixed, 12 overdentures) on 166 Brånemark implants in 32 older adults were compared with a matched group of prostheses on 162 implants in 34 younger subjects. Before loading, 9 implants failed in the older group and 13 in the younger. After loading, 3 failed in the older group and 10 in the younger.

Follow-up was from 4 to 17 yrs, and mean crestal bone loss was $0.2 \mathrm{~mm}$ in the first year after loading in both groups. Subsequently, the mean annual bone loss in both groups was 0.04 $\mathrm{mm}$ per yr. Data were insufficient for maxillary comparison, but there appeared to be greater bone loss with mandibular fixed prostheses in the younger group than in the older patients.

doi:10.1038/sj.bdj.4810663

\section{PERIODONTOLOGY}

\section{The effect of a triclosan-containing} dentifrice on the progression of periodontal disease in an adult population

Cullinan MP, Westerman B et al. J Clin Periodonto/ 2003; 30: 414-419

In a double-blind placebo controlled trial, the test dentifrice had a small additional effect on proximal pockets $>3 \mathrm{~mm}$.

A group of 504 subjects recruited from employees of a university was divided into 2 groups matched for periodontal disease, plaque level, age and gender. One group used a triclosan/ copolymer dentifrice, and the other, a placebo dentifrice. Subjects were examined for up to 5 yrs, and there was 19\% drop-out by that time. No other treatment was given, and subjects continued to receive care from their general dental practitioners.

Probing depth (PD) and attachment level measurements were made with an automated probe, and initial data analysis suggested that further attachment loss (AL) was delayed in subjects with pre-existing disease. There was no difference between groups in the basic parameter of AL, but there was an interaction between the active dentifrice and the number of sites $>3 \mathrm{~mm}$ PD at the previous examination which suggested that subjects with more such pockets at that time would have fewer pockets of that depth at the next examination than a subject using the placebo. 